\title{
FINITE ELEMENT IMPLEMENTATION OF CREEP CONSTITUTIVE MODEL INCLUDING TRANSIENT EFFECTS
}

\author{
D. Gabriel*, J. Masák*, J. Plešek* L. Kloc**, P. Dymáček**
}

\begin{abstract}
A complex creep constitutive model including transient effects was implemented in the finite element code PMD (Package for Machine Design). The material model for P-91-type creep-resistant steel together with computer implementation was verified by means of uniaxial stress loadings. Testing bar was discretized by the finite element method (FEM) and loaded with uniaxial stresses and constant temperatures that were used to demonstrate the analytical solutions in original paper.
\end{abstract}

Keywords: creep, transient effects, FEM.

\section{Introduction}

It is well known that creep experiments are time consuming and expensive. Moreover, the conventional creep tests are obviously available only for steady-state conditions while transient effects connected with varying conditions during startup or shutdown of the components are rarely explored experimentally. Instead, the transient phenomena seems to be an ideal field for computer modelling. The mathematical modelling of creep is usually based on the constitutive creep equations, which describe the strain rate dependence on stress, temperature and time, or creep strain, see Penny and Marriott (1971).

Numerical methods such as the finite element method (FEM) are apt tools for incorporating creep constitutive laws to describe the behaviour of real components under steady-state or transient conditions. The finite element method can be easily used to model components with more complicated geometry and serve for simulations of deformation and degradation processes for assessing life-limiting processes in such component parts. A relevant mathematical description of the material's properties under a wide range of stresses and temperatures is needed to obtain valid results. However, the current descriptions of the creep processes are based exclusively on the creep curves measured under constant loading conditions, so the transient effects caused by stress changes are ignored, see Kloc (2015).

Recently, complex model describing creep behaviour under variable stress conditions during primary and secondary creep stages was developed by Kloc et al. (2018). The model can handle transient effects on the stress changes, as well as low-stress creep behaviour. The constitutive equation was built using the relevant creep data for P-91-type steel, but it can be applied to the creep behaviour of most structural materials, e.g. the transient creep properties of the Sanicro 25 austenitic creep resistant steel were newly investigated in Kloc et al. (2019). In this work, the proposed creep material model including transient effects was implemented in the finite element code PMD-Package for Machine Design (2013). The material model together with computer implementation was verified by means of uniaxial stress loadings. Testing bar was discretized by the finite element method (FEM) and loaded with uniaxial stresses and constant temperatures that were used to demonstrate the analytical solutions in original paper Kloc et al. (2018).

Ing. Dušan Gabriel, Ph.D., Ing. Jan Masák, Ing. Jiří Plešek, CSc.: Institute of Thermomechanics of the Czech Academy of Sciences, v. v. i.; Dolejškova 1402/5; 182 00, Praha 8; CZ, \{gabriel, masak, plesek\}@it.cas.cz

** RNDr. Luboš Kloc, CSc., Ing. Petr Dymáček, Ph.D.: Institute of Physics of Material of the Czech Academy of

Sciences, v. v. i.; Žižkova 22; 616 62, Brno; CZ, \{kloc, pdymacek $\} @ i p m . c z$ 


\section{Complex creep phenomenological model including transient effects}

The complex material model developed by Kloc et al. (2018) is based on two distinct deformation mechanisms acting in parallel. The first mechanism, dominating at low stresses, is anelastic and it is based on a building of the field of internal stresses, assuming interaction of the hard elastic zones and soft elastoplastic zones. The other mechanism, dominating at higher stresses, is more obvious plastic deformation described by the modified Garofallo equation with threshold stress.

Since both mechanisms are assumed to be independent and running in parallel, the strain rate $\dot{\epsilon}$ can be expressed as the the sum of the high-stress mechanism rate $\dot{\epsilon}_{h}$ and the low-stress mechanism rate $\dot{\epsilon}_{l}$

$$
\dot{\epsilon}=\dot{\epsilon}_{h}+\dot{\epsilon}_{l}
$$

In high-stress region, the primary creep stage is assumed to be described by the $\mathrm{Li}$ equation proposed by $\mathrm{Li}$ (1963) in differential form. The strain rate $\dot{\epsilon}_{h}$ can be expressed as

$$
\dot{\epsilon}_{h}=\frac{\dot{\epsilon}_{s}\left(1+r_{i}\right)}{1+r_{i}-r_{i} \exp (-\theta)}
$$

where $\dot{\epsilon}_{s}$ is the secondary-stage creep rate, $r_{i}$ is a parameter characterising the ratio of the initial creep rate $\dot{\epsilon}_{i}$ to the secondary creep rate, $r_{i}=\dot{\epsilon}_{i} / \dot{\epsilon}_{s}-1$ and dimensionless variable $\theta$ describes the "creep age" of the material integrated according to

$$
\theta=\frac{1}{c} \int_{0}^{t}\left|\dot{\epsilon}_{s}\right| \mathrm{d} t
$$

where $t$ is time and $c$ is constant which describes the relation between the secondary stage creep rate and the primary relaxation time of the high-stress mechanism. The secondary-stage creep rate $\dot{\epsilon}_{s}$, in the equation (2) is described by the hyperbolic sine function (Garofalo-type equation)

$$
\dot{\epsilon}_{s}=\operatorname{sgn}(\sigma) b \exp \left(\frac{-Q_{h}}{R T}\right) \sinh \left(p\left(|\sigma|+\sqrt{\left(|\sigma|-\sigma_{t}\right)^{2}+\sigma_{r}^{2}}-\sqrt{\sigma_{t}^{2}+\sigma_{r}^{2}}\right)\right)
$$

where $\sigma$ is the applied stress, $\sigma_{t}$ is threshold stress, $\sigma_{r}$ describes the residual effective stress below the threshold, $Q_{h}$ is the apparent activation energy for the high-stress deformation mechanism, $R$ is the universal gas constant, $T$ is the absolute temperature, and $b$ and $p$ are parameters, where $b$ controls the overall rate of the high-stress mechanism and $p$ controls the transition between linear and exponential parts of the Garofallo equation.

For the low-stress region, the internal stress model from Kloc (2010) can be applied using a power-law relation with $n=3$. The strain rate of the low-stress mechanism $\dot{\epsilon}_{l}$ can be described as

$$
\dot{\epsilon}_{l}=\frac{\dot{\sigma}}{E}+\dot{\epsilon}_{c}
$$

where $E$ is Young's modulus and the strain rate $\dot{\epsilon}_{c}$ is given by

$$
\dot{\epsilon}_{c}=g \exp \left(\frac{-Q_{l}}{R T}\right)\left(\frac{\sigma}{\sigma_{t}}-\frac{(1-k) E}{k \sigma_{t}} \epsilon_{c}\right)^{3}
$$

where $Q_{l}$ is the apparent activation energy for the low-stress mechanism, and $g$ and $k$ are parameters, where $g$ controls the overall rate of the low-stress mechanism and $k$ describes the ratio between hard and soft zones elasticities. Note that the value of $\epsilon_{c}$ is obtained by numerical integration of equation (6). All parameters used to model the creep behaviour of the P-91-type steel are available in Kloc et al. (2018).

For the generalization of model into 3D some assumption must be adopted, see Kloc (2015): (i) the material is isotropic and remains isotropic, (ii) the creep strain is isochoric and (iii) the low-stress mechanism and the high-stress mechanism are independent even in 3D. From the third assumption above, the strain rate tensor $\dot{\varepsilon}$ can be expressed as

$$
\dot{\varepsilon}=\dot{\varepsilon}^{h}+\dot{\varepsilon}^{l}
$$


where $\dot{\varepsilon}^{h}$ and $\dot{\varepsilon}^{l}$ are strain rate tensors for high-stress and low-stress creep mechanisms, respectively. The high-stress part represented by equations (2)-(4), has a form of obvious creep equations, whose 3D generalisation procedure is based on the Prandtl-Reuss equations

$$
\dot{\varepsilon}^{h}=\frac{3}{2} \frac{\dot{\epsilon}_{c}^{h}}{\sigma_{e}} \mathbf{S}
$$

where $\mathbf{S}$ is deviatoric part of the $\boldsymbol{\sigma}$ tensor. The equivalent stress $\sigma_{e}$ is given by von Mises' function

$$
\sigma_{e}=\sqrt{\frac{3}{2} \mathbf{S} \cdot \mathbf{S}}
$$

The $\sigma_{e}$ is then used in equations (3),(4) and (2) to calculate equivalent creep rate $\dot{\epsilon}_{c}^{h}$ for the high-stress mechanism, which is applied in (7).

For the low-stress mechanism, 3D generalisation of the model yields

$$
\boldsymbol{\sigma}=\hat{C}\left(\varepsilon^{l}-\varepsilon^{c}\right)
$$

where $\varepsilon^{c}$ is the strain tensor of creeping element and $\hat{C}$ is fourth-order tensor of elasticity, which can be for isotropic material expressed in terms of $E$ and Poisson's ratio $\nu$. Similar procedure as applied for the high-stress mechanism can be used for the creeping element leading to equation for the strain rate of the low-stress creep mechanism

$$
\dot{\varepsilon}^{l}=\hat{C}^{-1} \dot{\boldsymbol{\sigma}}+\dot{\varepsilon}^{c}
$$

where the creep rate tensor $\dot{\varepsilon}^{c}$ can be obtained by similar way as $\dot{\varepsilon}^{h}$ in high-stress mechanisms, see details in Kloc et al. (2018).

Note that constitutive equations (7)-(11) are integrated by an original explicit algorithm for integration of differential systems arising from viscoplastic or creep finite element analyses, see Plešek and Korouš (2002). The algorithm combines Euler's forward scheme applied to discretised equilibrium equations at constant stress with a more precise procedure for the solution of local constitutive equations on the Gauss point level. Automatic subincrementation strategy ensures that the global error comprising both the truncation and roundoff errors remains bounded. The time step length is set on the basis of a posteriori error estimate.
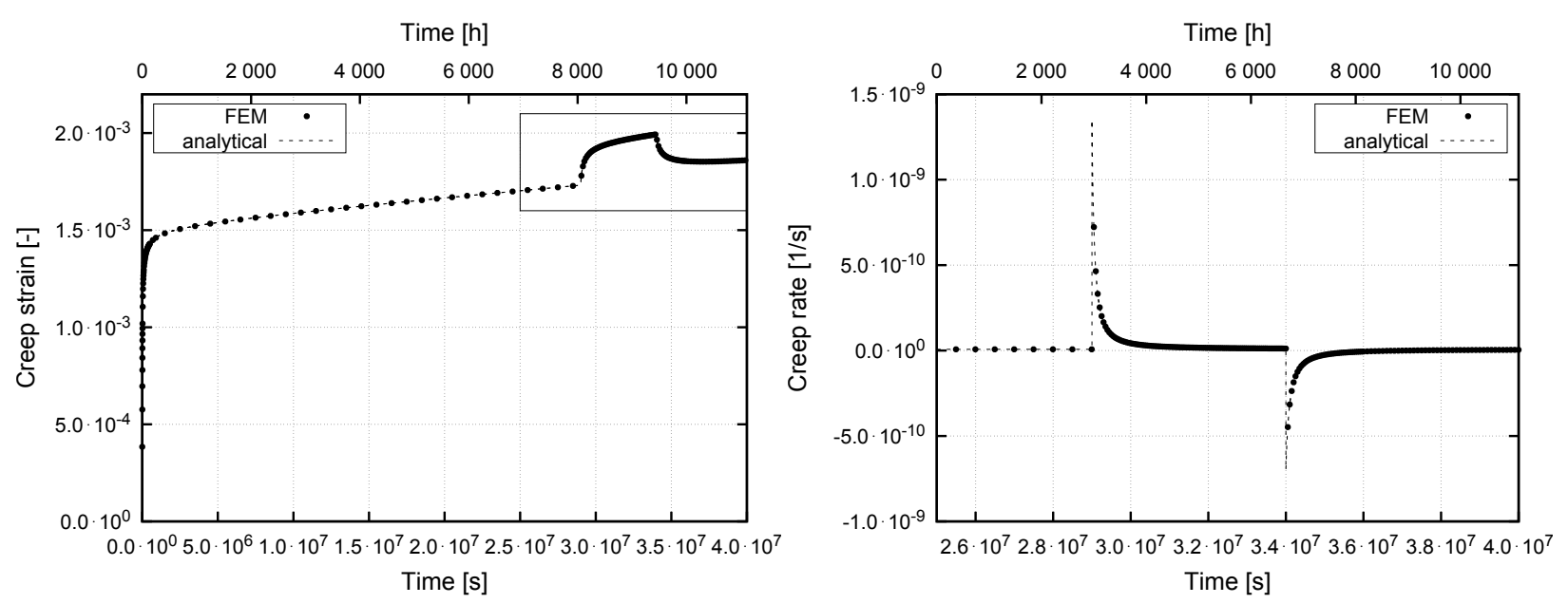

Fig. 1: Creep transient effect for P-91 steel upon stress change $\sigma=34.1 \rightarrow 39.4 \rightarrow 34.1 \mathrm{MPa}$ and temperature $T=600^{\circ} \mathrm{C}$ : creep strain distribution (left) and creep rate distribution for zoomed time interval when stress change occurred (right)

\section{Results}

The capability of the model to describe the transient creep behaviour for P-91 steel upon stress changes is demonstrated in Fig. 1. This well-know effect observed at low stress region is characterized by a large 
change in the creep rate as a result of changes in stress. The creep rate then returns slowly to an equilibrium value. Negative creep rate can be obtained after reducing the macroscopic stress even if the stress level remains positive. It should be noted that internal stress generated during the primary creep stage is considered to be responsible for this effect, see Kloc et al. (2018). Note that the transient effect behaviour is ignored completely with current creep constitutive equations because they are derived to describe only constant stress creep experiments.

Fig. 1 shows transient creep phenomena upon stress changes $\sigma=34.1 \rightarrow 39.4 \rightarrow 34.1 \mathrm{MPa}$ and temperature $T=600{ }^{\circ} \mathrm{C}$. The time distributions of creep strain (left) and creep rate (right) are compared for analytical (model) and FE solution. Note that distributions of creep rate are plotted for zoomed time interval when stress change occurred. The experimental data are available in original paper Kloc et al. (2018). It should be concluded that quite a good agreement between the analytical (model) and finite element solutions was observed.

\section{Acknowledgments}

The work was supported by the European Regional Development Fund under Grant No. CZ.02.1.01/0.0/0.0/ 15_003/0000493 (Centre of Excellence for Nonlinear Dynamic Behaviour of Advanced Materials in Engineering) and the Technology Agency of the Czech Republic under grant No TN01000024 (National Competence Center-Cybernetics and Artificial Intelligence)-sub-project "Automation and production system optimization" within institutional support RVO:61388998.

\section{References}

Penny, R. K. and Marriott, D. L. (1971) Design for Creep. McGraw.Hill, London.

Kloc, L. (2015) Critical View on the Creep Modelling Procedures. Acta Physica Polonica A, 128(4), pp 540-542. 13th International Symposium on Physics of Materials (ISPMA), Charles Univ, Fac Math \& Phys, Prague, Czech Republic, Aug 31-Sep 04, 2014.

Kloc, L., Sklenička, V., Dymáček, P., Plešek, J. (2018) New creep constitutive equation for finite element modelling including transient effects. Mechanics of Materials, 119, pp 49-55.

Kloc, L., Sklenička, V., Dymáček, P. (2019) Transient Effects in Creep of Sanicro 25 Austenitic Steel and Their Modelling. Metals, 9(2), No 245.

PMD version f77.11, VAMET/Institute of Thermomechanics, 2013, http://www.pmd-fem.com/.

Li, J. (1963) A Dislocation Mechanism of Transient Creep. Acta Metall., 11(11), pp 1269-\&.

Kloc, L. (2010) Internal stress model for pre-primary stage of low-stress creep. In: 15th International Conference on the Strength of Materials (ICSMA-15) (Skrotzki, W. and Oertel, CG. and Biermann, H. and Heilmaier, M. eds). Journal of Physics Conference Series, 240, 15th International Conference on the Strength of Materials (ICSMA 15), Dresden, Germany, Aug 16-21, 2009.

Plešek, J., Korouš, J. (2002) Explicit integration method with time step control for viscoplasticity and creep. Advances in Engineering Software, 33, pp 621-630. 